\title{
Predictive Factors for Conversion to Dementia in Individuals with Early-Onset Mild Cognitive Impairment
}

\author{
Kate Baird ${ }^{a}$ Sarah Baillon $^{b, c}$ Lilian Suh Lih Lau ${ }^{c}$ Mathew Storey ${ }^{d}$ \\ James Lindesay ${ }^{c}$ Latha Velayudhan ${ }^{a, c}$ \\ aDepartment of Old Age Psychiatry, Institute of Psychiatry, Psychology and Neurosciences, King's College London, \\ London, UK; ' beicestershire Partnership NHS Trust, Leicester, UK; ' ${ }^{\circ}$ Department of Health Sciences, University of \\ Leicester, Leicester, UK; dHull Royal Infirmary, Hull, UK
}

\section{Keywords}

Dementia - Mild cognitive impairment - Early onset · Risk factors $\cdot$ Predictors

\begin{abstract}
Introduction: There is little research on factors predicting conversion to dementia in early-onset mild cognitive impairment (eoMCl), a transitional stage between healthy ageing and dementia in individuals below the age of 65 . We aimed to examine whether sociodemographic and clinical factors at initial presentation predicted dementia progression in a cohort of eoMCl patients attending a memory service, at a university teaching hospital in the UK. Methods: This is a retrospective case note study of individuals diagnosed with eoMCI between 2000 and 2013 at the Younger Person's Memory Service (YPMS) in Leicestershire, England. Data collected at assessment included social factors, demographic characteristics, and medical and psychiatric history, as well as standardized cognitive assessment scores. Variables were analysed using $X^{2}$ or independent sample $t$ tests to identify associations. A Cox regression survival analysis was done to identify predictive factors for dementia conversion. An ROC analysis for total CAMCOG was used to investigate sensitivity and
\end{abstract}

karger@karger.com www.karger.com/dem

Karger $\stackrel{\text { ' }}{5}$

GOPEN ACCESS
(C) 2021 The Author(s)

Published by S. Karger AG, Basel

This is an Open Access article licensed under the Creative Commons Attribution-NonCommercial-4.0 International License (CC BY-NC) (http://www.karger.com/Services/OpenAccessLicense), applicable to the online version of the article only. Usage and distribution for commercial purposes requires written permission. specificity for dementia converters versus non-converters. Results: Out of 531 subjects who attended YPMS, 65 patients were given a diagnosis of eoMCl $(47.7 \%$ female; mean age $56.4 \pm 7.54$ years). Of these, 21 (32.3\%) converted to dementia during their course within the service. Comparison between subgroups revealed a significant association between dementia conversion and higher years of education and lower MMSE and CAMCOG (total and subscale) scores at baseline. Smoking history, alcohol use, or medical history such as diabetes or heart disease were not associated with conversion. Cox regression survival analysis showed higher education in years and lower total CAMCOG scores were significant predictors for conversion. Lower scores on the recent memory, remote memory, learning memory, and executive function subscales of the CAMCOG were also significant predictors for conversion. ROC curve analysis for total CAMCOG demonstrated that the best detection of dementia converters can be achieved with a cutoff score of 90.5/107 (sensitivity of $76.2 \%$ and specificity of $68.2 \%$ ). Area under the curve was 0.808 ( $95 \%$ Cl: 0.697-0.920). Conclusion: More years in education and lower cognitive scores on CAMCOG at initial assessment are associated with progression to dementia from eoMCl. Further research is required to explore these predictive factors more.

(c) 2021 The Author(s).

Published by S. Karger AG, Basel

Correspondence to:

Latha Velayudhan, latha.velayudhan@kcl.ac.uk 


\section{Introduction}

The majority ( $>97 \%$ ) of cases of dementia are late onset, with symptoms manifesting after 65 years of age. However, dementia also affects individuals who are younger than 65 years old (young-onset dementia) [1]. People with mild cognitive impairment (MCI) are individuals who suffer from mild deficits in cognition, but not enough to meet the criteria for a diagnosis of dementia, sometimes referred to as a form of prodromal dementia. These individuals have a greater risk of going on to develop dementia, with a conversion rate estimated around $10-15 \%$ per year, from initial assessment. However, a large number of individuals with MCI do not deteriorate further - and may even return to their baseline cognition [2]. Therefore, there may be risk factors that influence this conversion to dementia in MCI individuals.

The existing literature has identified various predictive factors for the conversion from MCI to dementia in older adults, such as APOE e4 status, vascular risk factors like hypertension, white matter lesions, and neuropsychiatric symptoms (such as depression) [3]. However, there is a limited amount of research on factors specifically predicting the conversion of $\mathrm{MCI}$ in those under the age of 65 years (early-onset MCI, eoMCI) to dementia [4]. In a group of 50 participants with eoMCI, those that converted to Alzheimer's within 2 years had a significantly higher atrophy rate of the left hippocampus, which was even faster in APOE e4 carriers [5]. Another study obtained CSF samples from 34 eoMCI patients and found that lower $A ß 42$ and higher $t$-tau levels have a greater risk of conversion to Alzheimer's at follow-up [6]. Scores from neuropsychological tests at initial assessment, such as language and memory (immediate and delayed), predicted conversion to Alzheimer's disease in 124 amnestic eoMCI patients [7], over a period of around 1.5 years. These studies were focused on conversion to Alzheimer's disease with limited follow-up, and the latter 2 studies compared risk of conversion in eoMCI with late-onset MCI (age at onset over 65 years). We therefore aimed to examine whether sociodemographic and clinical factors at the initial presentation predict dementia progression in a cohort of eoMCI patients attending a memory service, in a naturalistic setting at a university teaching hospital within the UK.

\section{Methods and Subjects}

This study included retrospective data of patients aged $<65$ years of age who were assessed at the Younger Person's Memory Service (YPMS) within the Mental Health Service for Older People at the Leicestershire Partnership National Health Service (NHS) Trust, UK, between January 2000 and December 2013, described previously [8]. Patients were referred by general practitioners from primary care and also specialists such as neurologists and general adult psychiatrists from secondary care, within Leicestershire and Rutland, East Midlands region of England. YPMS, a specialist memory clinic for younger adults, served a catchment of around 323,400 residents aged between 30 and 64 years as per ONS 2001 [9]. The study was approved by the local National Health Service Research Ethics Committee.

YPMS is a multidisciplinary team that provides assessment and management to all patients by a community psychiatric nurse, occupational therapist, psychologist, and psychiatrist [8]. The patients were assessed for cognitive functions, functional abilities, and behavioural symptoms in a 90-min semistructured interview. Everyone had neuroimaging or magnetic resonance imaging/computed tomography/single-photon emission computed tomography scan as appropriate, according to the local National Health Service (NHS) dementia assessment protocol, used routinely to assist diagnosis. At the end of the assessment, the team, led by the senior psychiatrist, met to arrive at a consensus diagnosis.

A standardized diagnosis for MCI (F06.7) and dementia with early onset was made according to ICD-10 criteria [10]. The core clinical criteria for MCI were in keeping with the National Institute on Aging-Alzheimer's Association (NIA-AA) criteria [11], that is, clinical and cognitive assessment was carried out following referral for cognitive concern reported by the patient, which are corroborated by a family member and observed by the clinician objectively on assessment, in the absence of functional decline and dementia. A subclassification of MCI, such as single or multi-domain, is generally not given in routine clinical assessments. Cognitive testing was carried out using the Mini-Mental State Examination (MMSE) and the Cambridge Cognition Examination (CAMCOG) part of the Cambridge Mental Disorders of the Elderly Examination (CAMDEX) [12, 13]. Baseline assessment data for those diagnosed with eoMCI were used for this study, which included psychosocial details with smoking and alcohol use, medical history, psychiatric history, cognitive tests, and behavioural assessments [8]. All patients diagnosed with eoMCI in the clinic were offered at least one annual review following diagnosis.

Statistical analysis was completed using SPSS software version 25. Patients who developed dementia during their course within the service were classified as "dementia converters," and those that did not as "non-converters." Independent sample $t$ tests and $\chi^{2}$ tests were used to compare these 2 groups. A $p$ value of 0.05 determined statistical significance. Missing values for cognitive measures were imputed with mean values for each group for the relevant measures. Variables were entered as potential covariates in a Cox regression survival analysis, using data only from patients followed up in the clinic. Clinical status $(1=$ converters, $0=$ nonconverters) was used as event status to define the conversion to dementia, and "duration of follow-up" was used as the time scale. In addition, receiver operating characteristic (ROC) analysis was done to investigate sensitivity and specificity of cutoffs for converters versus non-converters for the total CAMCOG. 
Table 1. Comparison of mean values for sociodemographic and cognitive variables between individuals with eoMCl who converted to a diagnosis of dementia (converters) and those who did not (non-converters)

\begin{tabular}{llll}
\hline Predictor variables & $\begin{array}{l}\text { Converters } \\
(n=21)\end{array}$ & $\begin{array}{l}\text { Non-converters } \\
(n=44)\end{array}$ & $p$ value \\
\hline Education, years & $13.50( \pm 2.37)$ & $11.29( \pm 2.29)$ & $0.001^{* *}$ \\
Gender (female) & $12(57)$ & $19(43)$ & 0.292 \\
Family history of dementia & $6(46)$ & $7(54)$ & 0.188 \\
Duration of illness at initial assessment, months & $17.45(17.398)$ & $44.56(75.543)$ & 0.186 \\
Age at first assessment & $57.14(5.85)$ & $56.43(8.00)$ & 0.899 \\
MMSE & $23.67( \pm 5.18)$ & $27.16( \pm 2.24)$ & $0.008^{* *}$ \\
Total CAMCOG & $80.14( \pm 12.34)$ & $91.80( \pm 5.42)$ & $0.000^{* *}$ \\
CAMCOG (orientation) & $8.52( \pm 1.91)$ & $9.59( \pm 0.66)$ & $0.006^{* *}$ \\
CAMCOG (comprehension) & $8.43(0.978)$ & $8.73(0.624)$ & 0.129 \\
CAMCOG (expression) & $17.48(2.358)$ & $18.48(2.151)$ & 0.060 \\
CAMCOG (remote memory) & $3.29( \pm 1.35)$ & $4.18( \pm 1.45)$ & 0.020 \\
CAMCOG (recent memory) & $3.14( \pm 1.06)$ & $3.66( \pm 0.71)$ & 0.019 \\
CAMCOG (learning memory) & $9.33( \pm 2.89)$ & $12.61( \pm 2.09)$ & $0.000^{* *}$ \\
CAMCOG (attention calculation) & $6.57( \pm 2.09)$ & $8.09( \pm 1.24)$ & $0.005^{* *}$ \\
CAMCOG (praxis) & $9.86( \pm 1.88)$ & $11.25( \pm 1.01)$ & $0.002^{* *}$ \\
CAMCOG (abstract thinking) & $6.19( \pm 1.37)$ & $6.89( \pm 1.10)$ & 0.040 \\
CAMCOG (perception) & $7.52(1.601)$ & $7.98(0.724)$ & 0.565 \\
CAMCOG (executive function) & $16.14( \pm 5.48)$ & $21.11( \pm 12.78)$ & 0.025 \\
\hline
\end{tabular}

Values are mean $( \pm \mathrm{SD})$ or $n(\%)$. eoMCl, early-onset mild cognitive impairment; MMSE, Mini-Mental State Examination; CAMCOG, Cambridge Cognition Examination total score and subscores. ${ }^{* *} p<0.01$.

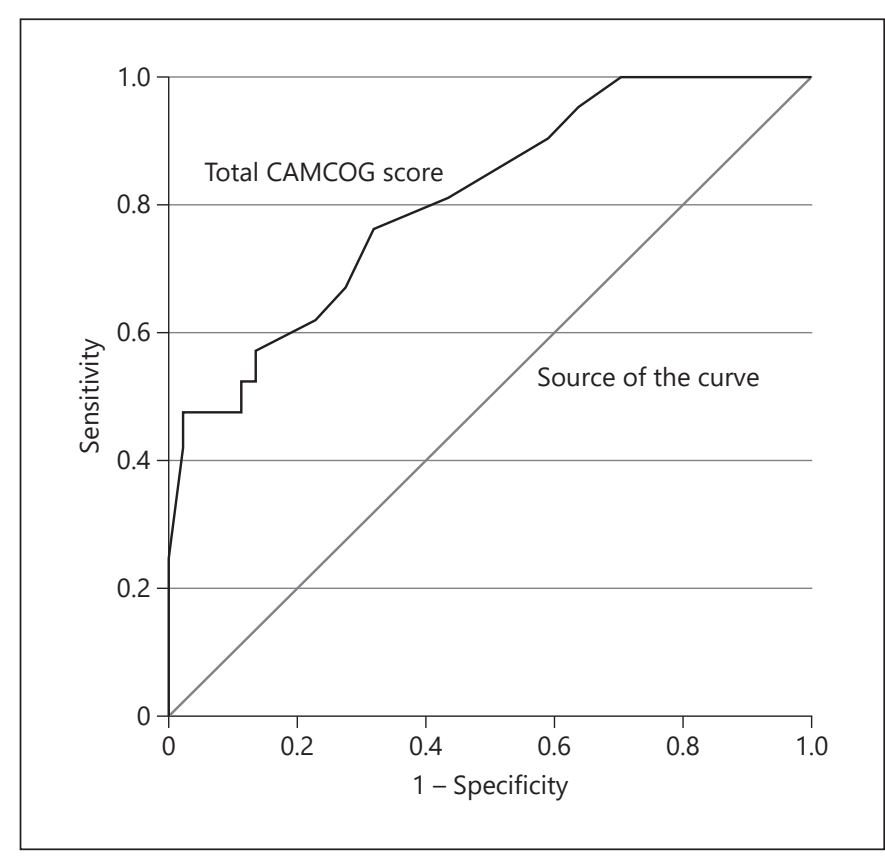

Fig. 1. ROC curve to investigate the sensitivity for converters and specificity for non-converters of the total CAMCOG assessment scores. ROC, receiver operating characteristic.

\section{Results}

Out of 531 subjects who attended YPMS between January 2000 and December 2013, 65 were given a diagnosis of eoMCI ( $47.7 \%$ female; age $56.4 \pm 7.54$ years). The mean duration of symptoms before presenting to the service was $35.37( \pm 63.29)$ months, with no difference between those who converted to dementia and those who did not ( $p=0.186)$. Of these, 21 patients $(32.3 \%)$ converted to dementia during their course within the service. The mean period of conversion was 30.40 months $( \pm 23.08)$.

Comparison between the subgroups revealed a significant association between higher years of education, lower MMSE, and specific CAMCOG (total and subscores) scores and converting to dementia (shown in Table 1). ROC curve analysis for total CAMCOG demonstrated that the best detection of dementia converters can be achieved with a cutoff point of 90.5/107 (sensitivity of $76.2 \%$ and specificity of $68.2 \%$ ) (shown in Fig. 1). Area under the curve was 0.808 (95\% CI: 0.697-0.920).

There was no significant association with gender $(p=$ $\left.0.292, \chi^{2}(1)=1.11\right)$, marital status $\left(p=0.465, \chi^{2}(4)=\right.$ $3.58)$, alcohol use $\left(p=0.617, \chi^{2}(4)=2.66\right)$, smoking status $\left(p=0.116, \chi^{2}(3)=5.90\right)$, or family history of dementia 
Table 2. Multivariate Cox regression results for predictors associated with change of clinical status (converters $=1$, non-converters $=0$ ) in patients with early-onset $\mathrm{MCl}$

\begin{tabular}{|c|c|c|c|c|}
\hline & Coefficient & Standard error & $p$ value & $\begin{array}{l}\mathrm{HR}(95 \% \mathrm{Cl}, \\
p \text { value) }\end{array}$ \\
\hline \multicolumn{5}{|l|}{ Model 1} \\
\hline Gender ( $0=$ female, $1=$ male $)$ & 0.90 & 0.56 & 0.106 & $2.57(0.83,7.38)$ \\
\hline Age, years (at first assessment) & 0.01 & 0.06 & 0.935 & $1.01(0.90,1.13)$ \\
\hline Education years & 0.31 & 0.12 & $0.013^{*}$ & $1.36(1.07,1.73)$ \\
\hline MMSE & -0.02 & 0.13 & 0.851 & $0.98(0.76,1.25)$ \\
\hline Total CAMCOG & -0.07 & 0.02 & $0.004^{* *}$ & $0.94(0.90,0.98)$ \\
\hline Family history of dementia & 0.16 & 0.58 & 0.777 & $1.18(0.38,3.67)$ \\
\hline \multicolumn{5}{|l|}{ Model 2} \\
\hline \multicolumn{5}{|l|}{ CAMCOG subscale scores } \\
\hline Orientation & 0.18 & 0.35 & 0.599 & $1.20(0.61,2.38)$ \\
\hline Comprehension & -0.49 & 0.54 & 0.362 & $0.61(0.22,1.75)$ \\
\hline Expression & 0.30 & 0.43 & 0.493 & $1.34(0.58,3.13)$ \\
\hline Recent memory & 1.27 & 0.49 & $0.009^{* *}$ & $3.54(1.37,9.19)$ \\
\hline Remote memory & -0.54 & 0.23 & $0.020 *$ & $0.59(0.37,0.92)$ \\
\hline Learning memory & -0.33 & 0.13 & $0.010^{*}$ & $0.72(0.56,0.92)$ \\
\hline Attention and calculation & 0.43 & 0.50 & 0.390 & $1.53(0.58,4.04)$ \\
\hline Praxis & -0.32 & 0.34 & 0.352 & $0.73(0.37,1.42)$ \\
\hline Abstract thinking & -0.32 & 0.38 & 0.396 & $0.72(0.34,1.53)$ \\
\hline Perception & 0.05 & 0.29 & 0.866 & $1.05(0.60,1.84)$ \\
\hline Executive function & -0.22 & 0.08 & $0.007^{* *}$ & $0.80(0.69,0.94)$ \\
\hline
\end{tabular}

$\mathrm{MCl}$, mild cognitive impairment; $\mathrm{HR}$, hazard ratio; $\mathrm{Cl}$, confidence interval; $\mathrm{MMSE}$, Mini-Mental State Examination; CAMCOG, Cambridge Cognition Examination. ${ }^{*} p<0.05 .{ }^{* *} p<0.01$.

$\left(p=0.188, \chi^{2}(1)=1.74\right)$. Past medical history (strokes, head injury, heart disease, diabetes, hypercholesterolaemia, and thyroid dysfunction) or mood symptoms (anxiety or depression) were also not significantly associated with the conversion of eoMCI to dementia.

One patient in the converter group had no information on follow-up data. Twenty-three out of 44 in the non-converter group were discharged the same day of baseline assessment and diagnosis, as they felt reassured, and did not come back for review. The Cox regression survival analysis showed that higher education in years and lower total CAMCOG score were significant predictors of the conversion from eoMCI to dementia (shown in Table 2, model 1). Lower scores on the recent memory, remote memory, learning memory, and executive function subscales of the CAMCOG assessment were also significant predictors for the conversion from eoMCI to dementia (shown in Table 2, model 2).

To explore the impact of single or multi-domain impairment on conversion to dementia, eoMCI patients were classified into amnestic versus non-amnestic eoMCI using a cutoff score of 18 or less for the memory component of the CAMCOG assessment [14]. 15 (71\%) patients with amnestic eoMCI and 6 (29\%) patients with non-amnestic eoMCI converted to dementia. Within the nonconverter group, 34 (77\%) had non-amnestic eoMCI and $10(23 \%)$ had amnestic eoMCI. Binary logistic regression analysis showed that non-amnestic eoMCI predicted dementia conversion (odds ratio $=8.50$ [95\% CI: $2.61-$ 27.68], $p<0.001)$.

\section{Discussion}

This study performed in routine clinical practice looked at risk factors for conversion to dementia in people with eoMCI. The study found more years in education and lower cognitive scores on CAMCOG at initial assessment are associated with those who progressed to dementia. Furthermore, specific lower CAMCOG subscale scores (recent memory, remote memory, learning memory, and executive function) also predicted disease progression to dementia in people with eoMCI. Factors such as smoking history, alcohol use, or significant medical history such as diabetes or heart disease were not significantly associated with dementia conversion. 
Our finding that dementia converters had higher education levels is a controversial finding. Although it is in keeping with a previous study that found converters had significantly higher mean years of education in comparison with non-converters [7], it contrasts with a recent report that suggests higher education as a protective factor for developing dementia [15]. A possible explanation for our results could be that individuals with higher levels of education demonstrate a faster cognitive decline compared to less educated individuals with similar levels of dementia severity, so present to services earlier [16]. Also, higher educated people may be more alert to the particular cognitive impairments that progress to dementia [17].

Scores on cognitive testing with MMSE and CAMCOG were significantly lower in the dementia converter group compared to the non-converters. In particular, the scores of the memory components of the CAMCOG assessment predicted conversion. Subscales such as the learning memory and remote memory look at declarative memory, conscious storing, and retrieval of data encoded in the brain. This type of memory has been frequently associated with being one of the earliest functions to be impaired in Alzheimer's, which could explain why these scores are significantly lower in young-onset dementia individuals [18]. These findings are similar to a previous study that found MMSE scores and memory components to be a predictor for conversion for Alzheimer's disease [7]. The total CAMCOG cutoff score of 90.5 demonstrates good sensitivity and specificity to identify dementia converters. This has important clinical value, to identify eoMCI patients who are at risk of converting to dementia.

We also attempted subcategorizing the participants into single-domain amnestic versus multi-domain eoM$\mathrm{CI}$ and found non-amnestic eoMCI predicted dementia conversion, contradicting our findings. This could possibly be due to overlap in items in CAMCOG, as the multi-domain score includes Verbal Fluency and Abstraction that not only measures executive function but also semantic memory (fluency) and intelligence (abstraction/similarities) and hence cannot validly be interpreted in isolation to determine whether people have memory deficits or not [19]. A more extensive neuropsychological examination of memory function that takes these methodological aspects into account is needed to examine single versus multi-domain eoMCI as predictors for dementia conversion in future studies.

Overall, the study has a number of strengths, the greatest being that it is a naturalistic study of data procured from a specialist clinical service over a period of 13 years, providing an extensive number of factors to be analysed. The longer time period these patients were followed up for surpasses previous research where the follow-up was much more restricted. However, there are also limitations to address. The sample size is small, and all participants were recruited from only one centre as an opportunistic sample. As prevalence rates of individuals with early-onset MCI have been stated to range from 0 to $13.7 \%$ per annum in clinical settings [20], a much larger sample size would be required to represent this population. As these are retrospective data from a clinical setting, as opposed to a research study, questionnaires such as for functional activity levels were not available for most patients for such analysis. Information on family history was obtained only from patients and family members, and APOE e4 status is not collected in routine clinical practice [21]. Data about APOE e4 status in future research would be useful. Similarly, clinical neuroimaging was done according to the local NHS trust dementia protocol during the period, and future studies would benefit from advanced neuroimaging and aetiology-based categorization of MCI [22]. Furthermore, not all patients in the non-converter group were followed up but were discharged on advice to return if they noticed any worsening in their cognitive or functional abilities. There are, however, chances of missing them if they moved away.

\section{Conclusions and Implications}

The present naturalistic study is one of the only one thus far that is conducted on a UK population and identifies potential variables that predict the likelihood of an individual with eoMCI converting to any type of dementia. This has vital clinical implications for the future, as identifying modifiable risk factors early allows preventative measures to be put in place to reduce the chance of conversion. Additional research is needed to explore these risk factors further, using data from a larger sample size that is more representative of a wider population.

\section{Acknowledgment}

The authors have no acknowledgements to disclose.

\section{Statement of Ethics}

The study was approved by the local National Health Service Research and Development Committee (Reference No. ELMH0583). 


\section{Conflict of Interest Statement}

The authors have no conflicts of interest to declare.

\section{Funding Sources}

This research did not receive any grants from funding agencies in the public, commercial, or not-for-profit sectors.

\section{Author Contributions}

L.V. designed the study and performed statistical analysis and interpretation. S.B., L.S.L.L., and M.S. contributed to the data collection. K.B. contributed to statistical analysis and wrote the manuscript with contributions from all authors.

\section{Data Availability Statement}

All data generated or analysed during this study are included in this article. Further enquiries can be directed to the corresponding author.

\section{References}

1 Cunningham EL, McGuinness B, Herron B, Passmore AP. Dementia. Ulster Med J. 2015; 84(2):79-87.

2 Alkhunizan M, Alkhenizan A, Basudan L. Prevalence of mild cognitive impairment and dementia in Saudi Arabia: a CommunityBased Study. Dement Geriatr Cogn Dis Extra. 2018;8(1):98-103.

3 Gao Q, Gwee X, Feng L, Nyunt MSZ, Feng L, Collinson SL, et al. Mild cognitive impairment reversion and progression: rates and predictors in community-living older persons in the Singapore longitudinal ageing studies cohort. Dement Geriatr Cogn Dis Extra. 2018; 8(2):226-37.

4 Roberts RO, Petersen RC. Predictors of earlyonset cognitive impairment. Brain. 2014; 137(5):1280-1.

5 Moon SW, Lee B, Choi YC. Changes in the hippocampal volume and shape in early-onset mild cognitive impairment. Psychiatry Investig. 2018;15(5):531-7.

6 Tábuas-Pereira M, Baldeiras I, Duro D, Santiago B, Ribeiro M, Leitão M, et al. Prognosis of early-onset vs. late-onset mild cognitive impairment: comparison of conversion rates and its predictors. Geriatrics. 2016;1(2):11.

7 Ye BS, Seo SW, Lee Y, Kim SY, Choi SH, Lee YM, et al. Neuropsychological performance and conversion to alzheimer's disease in early-compared to late-onset amnestic mild cognitive impairment: CREDOS study. Dement Geriatr Cogn Disord. 2012;34(3-4):156-66.

8 Velayudhan L, Baillon S, Urbaskova G, McCulloch L, Tromans S, Storey M, et al. Driving cessation in patients attending a young-onset dementia clinic: a Retrospective Cohort Study. Dement Geriatr Cogn Dis Extra. 2018; 8(1):190-8.

9 Office for National Statistics. Key Figures for 2001 Census: Key Statistics [Internet]. Census 2001. 2001.

10 World Health Organization. The ICD-10 classification of mental and behavioural disorders: Diagnostic criteria for research; 1993.

11 Albert MS, DeKosky ST, Dickson D, Dubois B, Feldman HH, Fox NC, et al. The diagnosis of mild cognitive impairment due to $\mathrm{Al}$ zheimer's disease: recommendations from the national Institute on Aging-Alzheimer's Association Workgroups on diagnostic guidelines for Alzheimer's disease. Focus. 2013;11(1):96-106.

12 Folstein MF, Folstein SE, McHugh PR. "Minimental state". A practical method for grading the cognitive state of patients for the clinician. J Psychiatr Res. 1975;12(3):189-98.

13 Huppert FA, Brayne C, Gill C, Paykel ES, Beardsall L. CAMCOG: a concise neuropsychological test to assist dementia diagnosissocio-demographic determinants in an elderly population sample. Br J Clin Psychol. 1995; 34(4):529-41.

14 Gallagher D, Mhaolain AN, Coen R, Walsh C, Kilroy D, Belinski K, et al. Detecting prodromal Alzheimer's disease in mild cognitive impairment: utility of the CAMCOG and other neuropsychological predictors. Int J Geriatr Psychiatry. 2010;25(12):1280-7.

15 Livingston G, Huntley J, Sommerlad A, Ames D, Ballard C, Banerjee S, et al. Dementia prevention, intervention, and care: 2020 report of the Lancet Commission. Lancet. 2020; 396(10248):413-46.

16 Contador I, Bermejo-Pareja F, Pablos DL, Villarejo A, Benito-León J. High education accelerates cognitive decline in dementia: a brief report from the population-based NEDICES cohort. Dement Neuropsychol. 2017;11(3):297-300.

17 Roe CM, Xiong C, Grant E, Miller JP, Morris JC. Education and reported onset of symptoms among individuals with Alzheimer disease. Arch Neurol. 2008;65(1):108-11.

18 Jahn H. Memory loss in Alzheimer's disease. Dialogues Clin Neurosci. 2013;15(4):445-54.

19 Kessels RPC, Mimpen G, Melis R, Olde Rikkert MGM. Measuring impairments in memory and executive function in older people using the revised Cambridge cognitive examination. Am J Geriatr Psychiatry. 2009;17(9): 793-801.

20 Kremen WS, Jak AJ, Panizzon MS, Spoon KM, Franz CE, Thompson WK, et al. Early identification and heritability of mild cognitive impairment. Int J Epidemiol. 2014;43(2): $600-10$.

21 Velayudhan L, Baillon S, Daby L, Suntharamoorthy P, Kablan A, Tromans S, et al. Predictors of disease progression in early-onset Alzheimer's dementia: a Retrospective Cohort Study. J Am Med Dir Assoc. 2020;21(11): 1735-9.

22 Rallabandi VPS, Tulpule K, Gattu M. Automatic classification of cognitively normal, mild cognitive impairment and Alzheimer's disease using structural MRI analysis. Inform Med Unlocked. 2020;18:100305. 